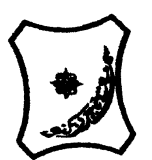

Bayero Journal of Pure and Applied Sciences, 12(1): 337 - 343

ISSN $2006-6996$

\title{
OPTIMIZATION OF LEVULINIC ACID PRODUCTION FROM GROUNDNUT SHELL USING TAGUCHI ORTHOGONAL ARRAY DESIGN
}

\author{
Halliru, A., ${ }^{1 *}$, Hassan, L.G. ${ }^{2}$ and Muhammad, A. S. ${ }^{2}$ \\ ${ }^{1}$ School of secondary Education Sciences, Department of Chemistry, Federal College of Education, \\ Katsina. \\ ${ }^{2}$ Department of Pure and Applied Chemistry, Faculty of Sciences, Usmanu Danfodiyo University, \\ Sokoto. \\ Ighassan2002@yahoo.com; rafindadi2008@yahoo.com
}

\begin{abstract}
The exploitation of lignocellulosic biomass is receiving an increasing attention due to its renewability, abundance and low price value, and can be converted into various valuable platform compounds such as furfural, lactic acid, formic acid and levulinic acid. Among these products, levulinic acid (LA) is the main compound of biomass hydrolysis, which has been classified by the United States Department of Energy as one of the top-12 promising building blocks. This work reported the transformation of groundnut shell into $L A$. The production of $L A$ was carried out in a $50 \mathrm{~cm}^{3}$ Teflon lined stainless steel reactor. The $L A$ produced was extracted from the aqueous mixture using ethyl acetate, about $1 \mathrm{~g}$ of sodium sulphate anhydrous were added to remove the water in the organic layer after the aqueous layer was drained and then heated at a temperature of about $78{ }^{\circ} \mathrm{C}$ for the solvent to evaporate and $L A$ was the residue. The production process was optimized using a Taguchi orthogonal array design, with optimum yield of $74.54 \%$ at reaction conditions of temperature $\left(180^{\circ} \mathrm{C}\right)$, time $(3.5 \mathrm{~h})$, and acid concentration $(0.3 \mathrm{M})$. The FTIR spectrum of the produced LA showed absorption at about $1705.13 \mathrm{~cm}^{-1}$ and 3039.91 $\mathrm{cm}^{-1}$ indicating the conjugated carbonyl and the hydroxyl of carboxylic acid functional group. It was recommended that high yields of $L A$ can be achieved across a range of optimization variables as long as two out of the three conditions are met: high acid catalyst concentration, long reaction time or high temperature within the range tested, as $L A$ is relatively stable once formed. Moreover, the results obtained revealed that groundnut shell could be a potential substrate for levulinic acid production.
\end{abstract}

Keywords: Groundnut shell, Levulinic acid, Optimization, Hydrolysis, Taguchi design.

\section{INTRODUCTION}

The continuous depletion of fossil fuel resources and increasing global energy consumption have led to considerable attention on the utilization of lignocellulosic biomass for production of chemicals, fuels, and energy (Cirujana et al., 2015). Levulinic acid (LA) is lignocellulosic biomass derived chemical and regarded as one of the top twelve building block chemicals (Ramli and Amin, 2015). One of the main concerns of biochemical production is the consumption of edible biomass such as corn, sugarcane, soya beans, and vegetables as feedstock for biofuels generation. This issue has motivated researchers around the world to develop second-generation<smiles>CC(=O)CCC(=O)O</smiles>

Figure 1: The Molecular Structure of Levulinic Acid technologies for processing non-edible biomass (lignocellulosic materials) so that a sustainable production of a new generation of fuels and chemicals can be achieved without affecting food supplies.

Levulinic acid (LA) also known as 4oxopentanoic acid or 4-ketopentanoinc acid is a linear $\mathrm{C}_{5}$-alkyl carbon chain. Levulinic acid is a widely used industrial chemical with one carbonyl, one carboxyl, and $\alpha-\mathrm{H}$ in its inner structure (Figure 1), which belongs to a short chain and non-volatile fatty acid (Darenberg et al., 2011). 
Lignocellulosic biomass is the most abundant biomass in the world which can be converted into various valuable platform compounds such as furfural, lactic acid, formic acid and levulinic acid by biorefinery processes (Elumaila et al., 2016). Among these compounds, levulinic acid (LA), has wide applications in industry and agriculture to produce a variety of products such as plasticizer, oil additives and fragrances (Yuan et al., 2016). Up to now, the production of LA has been broadly investigated from a variety of feedstock such as sugars, cellulose, chitin and raw lignocellulosic biomass by homogeneous or heterogeneous catalysts (Sun et al., 2016). Compared with homogeneous mineral acid catalysts such as $\mathrm{HCl}, \mathrm{H}_{2} \mathrm{SO}_{4}$ and $\mathrm{H}_{3} \mathrm{PO}_{4}$, heterogeneous catalysts such as acidic ionexchange resins, heteropoly acids, and metal oxides can be easily isolated from the reaction mixture and reused, and they have no corrosion problem. However, heterogeneous catalysts are still not satisfactory in LA production, especially from cellulose and raw biomass feedstock, due to the solid-solid mass transfer limitation (Antonetti et al., 2016).

Therefore, although mineral acids have the disadvantages of equipment corrosion and difficulty in recycling, they have been broadly used in producing LA and are beneficial for industrial-scale production because of their high activity and low cost (Bevilaqua et al., 2013). In addition, the corrosion problem of the equipment can be partially avoided by the employment of dilute acid or acid-resistant materials (Antonetti et al., 2016). In terms of starting materials used for LA production, monosaccharides such as glucose and fructose can give high LA yields (Upare et al., 2013). The high cost of sugars restricts their application in LA production. However, the production of LA from cellulose and in particular, from raw and waste biomasses is preferred because of their abundance and low cost. When cellulose or raw lignocellulosic biomass are used as starting materials, LA is usually produced by the acidcatalyzed conversion of cellulose, following a reaction pathway in which cellulose is hydrolyzed into glucose via enzymatic or acid-catalysis methods and the generated glucose is dehydrated to 5-hydroxymethylfurfural (HMF), followed by a rehydration step converting HMF to $L A$, as shown in Figure 2 (Elumaila et al., 2016).

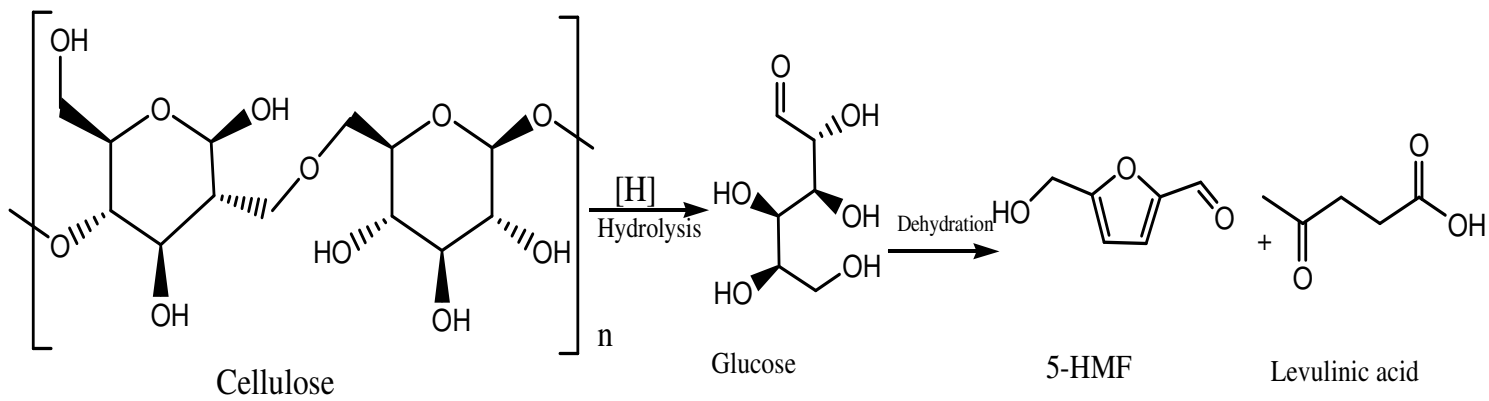

Figure 2: Reaction Pathway for the Acid-catalyzed Conversion of Cellulose to Levulinic Acid

The objectives of this study are to explore the potential of groundnut shell as feedstock for levulinic acid production and optimize the reaction parameters (acid concentration, temperature, and reaction time) via simultaneous hydrolysis and dehydration.

\section{MATERIAL AND METHODS}

Groundnut shells were collected from the processing sites at Adarawa village behind Usmanu Danfodiyo University, Sokoto stadium. The sample was identified as groundnut shell (Arachis hypogaea shell) at the Crop Science Department, Faculty of Agriculture, Usmanu Danfodiyo University, Sokoto. The shells were crushed, milled and sieved. Particles that passed through the $40 \mathrm{~mm}$ mesh sieve were retained and stored in a plastic container until required for the analysis

\section{Method}

The methods described by Mosier et al. (2015) and Kuhad et al., (2010) was adopted for the extraction of cellulose from dried sample as follows:

\section{Acid Pretreatment}

The dried groundnut shell $(3.0 \mathrm{~g})$ was pretreated with $3 \%(\mathrm{v} / \mathrm{v})$ hydrochloric acid (30 $\mathrm{cm}^{3}$ ) for 30 minutes in an autoclave at $121^{\circ} \mathrm{C}$. The content was cooled to ambient temperature, then filtered, washed to a neutral $\mathrm{pH}$ and dried overnight at a temperature of $50{ }^{\circ} \mathrm{C}$ in an oven. This was aimed to remove the hemicelluloses. 
Special Conference Edition, November, 2019 Alkali Pretreatment

The oven dried substrate $(2.0 \mathrm{~g})$ from acid pretreatment was further pretreated with $4 \%(\mathrm{w} / \mathrm{v})$ sodium hydroxide $\left(20 \mathrm{~cm}^{3}\right)$ for 15 minutes in an autoclave at $121{ }^{\circ} \mathrm{C}$ to remove lignin. The solution was cooled, filtered, washed to a neutral $\mathrm{pH}$ and oven dried overnight at a temperature of $50{ }^{\circ} \mathrm{C}$

\section{Cellulose Test}

The method described by Achor et al., (2014) was adopted with modification. Briefly, about 0.1 $g$ Cellulose powder was placed on a watch glass, and dispersed in a drop of iodinated zinc chloride solution and the color change was observed. The cellulose extracted from groundnut shell was used for the production of levulinic acid.

\section{Lignin Test (Wiesner Test)}

The oven dried substrate $(1.0 \mathrm{~g})$ from alkali pretreatment was dissolved with few drops of dilute hydrochloric acid on a glass slide. Two drops of benzene-1,3,5-triol (phloroglucinol) were added and the slide was slowly heated over a bunsen burner, until the liquid content was evaporated. The slide was then examined under a light microscope, (Achor et al., 2014)

\section{Levulinic acid Production}

The synthetic reaction was carried out in a 50 $\mathrm{cm}^{3}$ Teflon lined stainless steel reactor. $2.0 \mathrm{~g}$ of pretreated sample was placed in $20 \mathrm{~cm}^{3}$ of $0.2 \mathrm{M}$ $\mathrm{HCl}$ aqueous solution and loaded into the reactor. The reactor was immersed in the paraffin oil bath. Then the closed reactor was magnetically stirred at $1000 \mathrm{rpm}$ and heated to $180^{\circ} \mathrm{C}$ using hot plate magnetic stirrer and kept for 3.5 hours. After the reaction, the reactor was quenched with cold water to stop the reaction and a liquid sample after filtration was obtained, (Jialei et al., 2017).

The levulinic acid produced was extracted from the aqueous mixture; equal volume of ethyl acetate to the levulinic acid mixture was placed in a separating funnel; two layers were obtained: an organic and aqueous layer, the aqueous layer was drained and the organic layer was placed in a beaker and approximately $1.0 \mathrm{~g}$ of sodium sulphate anhydrous was added to extract water in the organic layer, then placed in a fume cupboard and heated at a temperature of about $78{ }^{\circ} \mathrm{C}$ to evaporate the solvent and LA was the residue (Jialei et al., 2017).

\section{Optimization of Levulinic acid Production Process}

The optimization of levulinic acid production was conducted using Minitab 17 statistical software, comparing parameters such as temperature, time and acid concentration to determine which parameters will produce a higher LA yield. The Taguchi orthogonal array design was done to determine the optimum conditions for the production of levulinic acid. Parameters that were used for optimization settings are given in Table 1, after inserting the minimum and maximum of the three parameters, a set of experiments were given from the software and Table 2 shows the experiments that needed to be done to measure the amount of LA produced. The pretreated substrate was used as a starting material for this experiments and dilute $\mathrm{HCl}$ was used as a catalyst using a Teflon lined stainless steel reactor with a $50 \mathrm{~cm}^{3}$ reaction volume and 70 bar pressure.

Table 1: Experimental Range and Levels of Independent Variables

\begin{tabular}{lll}
\hline Parameter & Minimum & Maximum \\
\hline Temperature $\left({ }^{\circ} \mathrm{C}\right)$ & 160 & 220 \\
Reaction Time (Hours) & 2.0 & 3.5 \\
Acid Conc. (M) & 0.2 & 0.35 \\
\hline
\end{tabular}

The ranges of variables were chosen based on information from a previous study (Jialei et al., 2017). The effects of temperature, time and acid concentration that affect the hydrolysis and dehydration stage were optimized and the hexose sugars in the extracted cellulose were used as the substrate for levulinic acid production. Temperature $\left(160-220{ }^{\circ} \mathrm{C}\right)$, residence time (2.0 - 3.5 hours) and acid concentrations $(0.2-0.35 \mathrm{M})$ were selected as independent process variables. 16 treatments were run in a random order to minimize the effect of any unexplained variability in the observed responses. The response measured was the yields of levulinic acid. 
Special Conference Edition, November, 2019

RESULTS AND DISCUSSION

Table 2 and 3 shows the results of physicochemical properties of cellulose extracted from groundnut shell and taguchi orthogonal array design for LA yield.

\section{Table 2: Physicochemical Properties of Cellulose Extracted from Groundnut Shell}

\begin{tabular}{lll}
\hline Test & Observation & Inference \\
\hline $\begin{array}{c}\text { (i) Cellulose powder } \\
+ \text { iodinated } \mathrm{ZnCl}_{2}\end{array}$ & Turns violet blue & Cellulose is present \\
$\begin{array}{c}\text { (ii) Cellulose powder }+ \text { dilute } \mathrm{HCl} \\
+ \text { benzene-1,3,5-triol }\end{array}$ & No reaction & Lignin is absent \\
\begin{tabular}{l} 
(iii) $\mathrm{pH}$ \\
\hline
\end{tabular} & 6.9 & \\
\hline
\end{tabular}

The optimization of LA was carried out based on the three factors variables (reaction temperature, reaction time and acid concentrations) which are in four different levels of the experimental runs. From the set of experiments, it showed that the acid hydrolysis of pretreated groundnut shell with $0.30 \mathrm{M}$ at 180 ${ }^{\circ} \mathrm{C}$, for 3.5 hours gave the highest yield of 74.54 $\%$ LA compared with other parameters (Table 3 ), these are the optimum parameters used for the LA synthesis.

Table 3: Taguchi Orthogonal Array Design and LA Yield

\begin{tabular}{lllll}
\hline Run. & TEMP. $\left({ }^{\circ} \mathrm{C}\right)$ & TIME $(\mathrm{H})$ & ACID CONC. (M). & LA YIELD (\%) \\
\hline 1 & 160 & 2.0 & 0.20 & 33.56 \\
2 & 160 & 2.5 & 0.25 & 46.25 \\
3 & 160 & 3.0 & 0.30 & 31.45 \\
4 & 160 & 3.5 & 0.35 & 40.10 \\
5 & 180 & 2.0 & 0.25 & 52.74 \\
6 & 180 & 2.5 & 0.20 & 60.37 \\
7 & 180 & 3.0 & 0.35 & 63.42 \\
8 & 180 & 3.5 & 0.30 & 74.54 \\
9 & 200 & 2.0 & 0.30 & 28.32 \\
10 & 200 & 2.5 & 0.35 & 31.46 \\
11 & 200 & 3.0 & 0.20 & 26.32 \\
12 & 200 & 3.5 & 0.25 & 41.65 \\
13 & 220 & 2.0 & 0.35 & 17.48 \\
14 & 220 & 2.5 & 0.30 & 25.20 \\
15 & 220 & 3.0 & 0.25 & 33.25 \\
16 & 220 & 3.5 & 0.20 & 29.62 \\
\hline
\end{tabular}

The general linear model analysis was carried out to fit the response variable and to investigate the variable that is significant. The ' $P$ ' values for all the factors used were less than 0.05 this indicated that all the terms are statistically significant. The correlation coefficient $\left(R^{2}\right)$ of the analysis was found to be $96.50 \%$ which shows that the variables are significant and hence fit this model.

\section{Identification of Levulinic acid}

Table 4 shows the characteristics FTIR peaks of the characterized levulinic acid. The produced levulinic acid was identified using Shimadzu8400s Fourier Transform Infrared Spectrophotometer (FTIR) at $4000-750 \mathrm{~cm}^{-1}$ transmission rate. 
Special Conference Edition, November, 2019

Table 4: FT-IR Absorption Bands of Levulinic Acid Produced

\begin{tabular}{lll}
\hline Frequency $\left(\mathrm{cm}^{-1}\right)$ & Bond & Functional group \\
\hline $3039.91(m$ and $b)$ & O-H stretch & Hydroxyl \\
$2939.61(m)$ & C-H stretch & C-H \\
$1705.13(s)$ & C=O stretch & C=O (Carbonyl) \\
$1751.42(w)$ & C=O stretch & C=O (Carbonyl) \\
$1226.77(m)$ & C-O stretch & C-O \\
\hline
\end{tabular}

Where $(m)$ - medium peak, $(s)$-sharp peak, $(w)$ - weak peak and $(b)$ broad peak

The important vibrations of LA are all seen in this FTIR spectrum, vibration such as $\mathrm{O}-\mathrm{H}$ $\left(3039.91 \mathrm{~cm}^{-1}\right.$ ) which is broad and intense stretching for carboxylic group, a very strong absorption at $1705.13 \mathrm{~cm}^{-1}$ which corresponds to absorption of carbonyl $(\mathrm{C}=\mathrm{O})$ stretching for ketone of the carboxylic group, and $\mathrm{C}-\mathrm{H}$ $\left(2939.61 \mathrm{~cm}^{-1}\right)$ stretching for alkane was observed in this spectrum. The $\mathrm{sp}^{2}$ hybridized C$\mathrm{O}$ vibration stretching for carboxylic group which absorbs around $1226.77 \mathrm{~cm}^{-1}$ differentiates the $\mathrm{C}-\mathrm{O}$ stretching for alcohol an $\mathrm{sp}^{3}$ hybridized that absorbs around $1000-1150 \mathrm{~cm}^{-1}$. Another thing that differentiates this spectrum from that of alcohol is the broad $\mathrm{O}-\mathrm{H}$ stretching, both carboxylic acid and alcohol hence these broad absorptions but the $\mathrm{O}-\mathrm{H}$ vibration stretching for carboxyl group absorbs around $2500-3300 \mathrm{~cm}^{-1}$ whereas the $\mathrm{O}-\mathrm{H}$ vibration stretching for alcohols absorbs between $3200-3500 \mathrm{~cm}^{-1}$ and these have confirmed the presence of carboxyl group in the spectrum as shown in Figure 3.

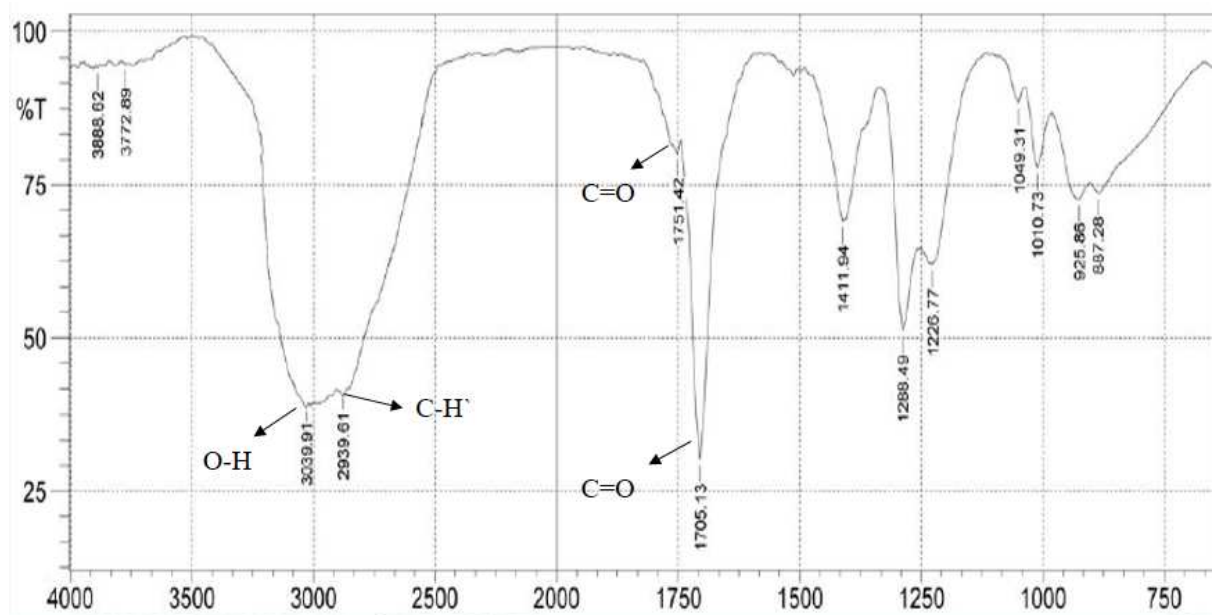

Figure 3: FT-IR Spectrum of LA produced from Groundnut shell

Effect of Reaction Temperature on the Synthesis of LA

In the catalytic conversion of lignocellulosic biomass, the reaction temperature is an important effect factor that plays a crucial role in determining the products' distribution (Yuan et al., 2016). LA is generally produced by the rehydration of 5-hydroxymethylfurfural (Rout et al., 2016), which is obtained by the dehydration of glucose or fructose through eliminating three water molecules (Fachiri et al., 2015). The hydrolysis of cellulose to glucose is considered to be a key step in the production of LA directly from lignocellulosic biomass (Huang and Fu, 2013). Generally, the production of levulinic acid requires higher temperatures as compared with the hydrolysis of cellulose (Sun et al., 2016). The influence of the reaction temperature on the synthesis of LA from pretreated groundnut shell was investigated (Figure 4). When the reaction was conducted at $160{ }^{\circ} \mathrm{C}$, the LA yield increased slowly with the increasing reaction time, and a LA yield of $51.45 \%$ was attained in a 3.0 hours' $^{\prime}$ reaction time. When the temperature was increased to $180{ }^{\circ} \mathrm{C}$ the LA yields increased rapidly from $51.45 \%$ to $74.74 \%$ between 2.0 to

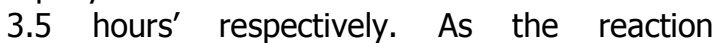
temperature increased from $200{ }^{\circ} \mathrm{C}$ to $220{ }^{\circ} \mathrm{C}$ a decreased in LA yield with time was observed compared to a temperature of $180^{\circ} \mathrm{C}$. Thus, 180 ${ }^{\circ} \mathrm{C}$ was considered an optimum temperature for the production of LA from groundnut shell. 


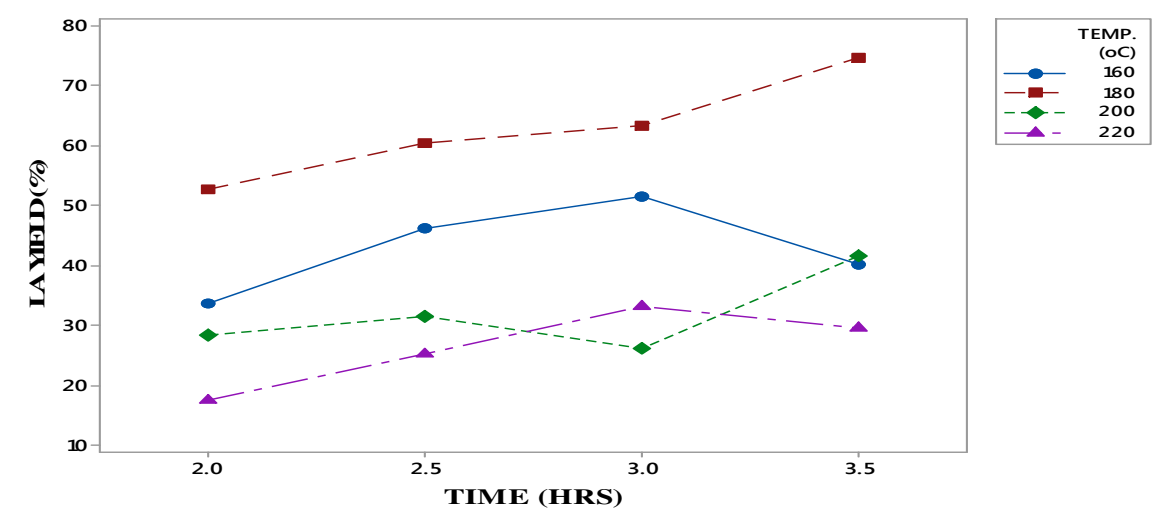

Figure 4: Interaction Plot Showing Effect of Reaction Temperature on the Synthesis of LA.

\section{Effect of Acid Concentration on the Synthesis of LA}

The effect of the acid concentration on the catalytic synthesis of LA from the pretreated groundnut shell was investigated (Figure 5). When the reaction was performed in $0.2 \mathrm{M} \mathrm{HCl}$ aqueous solution, the LA yield increased rapidly with the prolonged reaction time, and LA yield of $60.37 \%$ was achieved at 2.5 hours' reaction time and decreased to $26.32 \%$ at 3.0 hours. With increased in $\mathrm{HCl}$ concentration, the LA yield significantly increased, and maximum LA yields of $74.54 \%$ at 3.5 hours and $63.42 \%$ at 3.0 hours were obtained for $0.3 \mathrm{M}$ and $0.35 \mathrm{M} \mathrm{HCl}$ aqueous solutions, respectively.

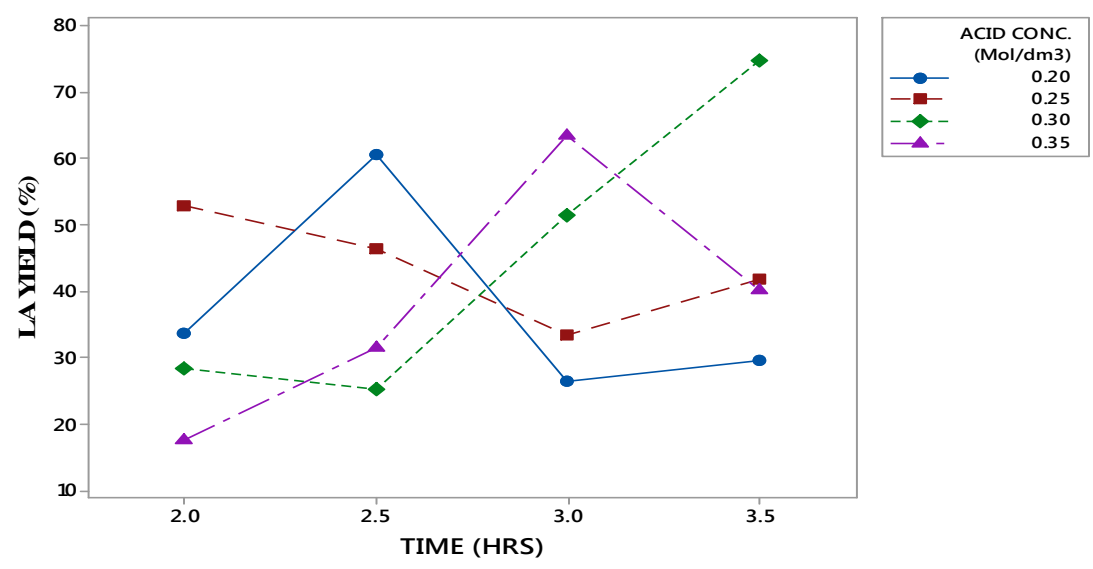

Figure 5: Interaction Plot Showing Effect of Acid Concentration on the Synthesis of LA.

\section{CONCLUSION}

The production of chemicals from biomass represents a major challenge because of the complex nature of the biomass substrate and non-cellulose components. Product yields and process viability can be enhanced if the hemicellulose and lignin components are removed. This study illustrated that levulinic acid production from groundnut shell and optimization of process variables were performed using one-stage process. Maximum levulinic acid yield $(74.54 \%)$ was obtained at optimum conditions of temperature $\left(180^{\circ} \mathrm{C}\right)$, time (3.5 hours) and acid concentration of ( 0.3
M). From the result obtained, it can be concluded that groundnut shell could be a potential raw material for the production of a bio-based chemical such as levulinic acid. High yields of levulinic acid can be achieved across a range of optimization variables as long as two out of the three conditions are met: high acid catalyst concentration, long reaction time or high temperature within the range tested, as levulinic acid is relatively stable once formed. However, increasing the temperature to $200{ }^{\circ} \mathrm{C}$ shows a decrease in LA yield with increasing reaction time. 
Special Conference Edition, November, 2019 REFERENCES

Achor, M. Oyeniyi, Y. J. and Yahaya A. (2014) Extraction and Characterization of Microcrystalline Cellulose obtained from the back of Lagerriana siceraria (water guurd). Applied Pharmaceutical Science. 4(1), 57-60.

Antonetti, C., Licursi, D., Fulignati, S., Valentini, G., and Respolli, G. A. (2016). New Frontiers in the Catalytic Synthesis of Levulinic Acid: From Sugars to Raw and Waste Biomass as Starting Feedstock. Catalysts, 6(196).

Bevilaqua, D. B., Rambo M, K. D., Rizetti, T. M., Cardoso, A. L., and Martins, A. F. (2013). Cleaner Production: Levulinic Acid from Rice Husks. Cleaner products, 47, 96-101.

Cirujana, F. G., Corma, A., Llabrus, I., and Xamana, F. X. (2015). Conversion Of Levulinic Acid Into Chemicals: Synthesis Of Biomass-Derived Levulinate Esters Over $\mathrm{Zr}$ Containing MOF3. Chemical Engineering Science, 124, 52-60.

Darenberg, G., Gerhardi, M., and Kamm, B. (2011). Biobased Fuel and Additives from Lignocellulose Feedstocks Via the Production of Levulinic Acid and Furfural. Chemical Industrial Program, $65,439-451$.

Elumaila, S., Agarwal, B., Runge, T. M., and Sungwan, R. S. (2016). Integrated Twostage Chemically Processing of Rice Straw Cellulose to Butyl Levulinate. Carbohydrate Polyner, 150, 286-298.

Fachiri, B. A., Abdilla, R. M., Van de Brovenkamp, H. H., Resrendra, C. B., and Heenes, H. J. (2015). Experimental and Kinetic Modeling Studies on the Sulfuric Acid-Catalyzed Conversion of Dfructose to 5-Hydroxymethylfurfural and Levulinic Acid in Water. ACS Sustainable Chemical Engineering, 3, 3024-3024.

Huang, Y. B., and Fu, Y. (2013). Hydrolysis of Cellulose to Glucose by Solid Acid Catalysts. Green Chemicals, 15, 10951111.
Jialei, S., Feng, S., Mo, Q., and Xinhuu, Q. (2017). High-Yield Production of Levulinic Acid From Pretreated Cow Dung in Dilute Acid Aqueous Solution. Molecule, 2, 1-9.

Kuhad, R. C., Gupta, R., Khasa, Y. P., and Singh, A. (2010). Bioethanol Production From Lantana comara (Red Saga): Pretreatment, Saccharification And Fermentation. Bioresouce Technology, 101, 8348-8354.

Mosier, N., Wyman, C., and Dale, B. (2015). Features Of Promising Technologies For Pretreatment Of Lignocellulosic Biomass. Bioresource Technology, 96, 673-686.

Ramli, N. A., and Amin, N. A. (2015). Optimization of Renewable Levulinic Acid Production From Glucose Conversion Catalysed By Fe/HY Zeolite Catalyst In Aqueous Medium.Energy Conversion And Management, 95, 1019.

Rout, P. K., Nannaware, A. D., Prakash, O., Kalra, A., and Rajesekharan, R. (2016). Synthesis of Hydroxymethylfurfural from Cellulose Using Green Processes: A Promising Biochemical and Biofuel Feedstock. Chemical Engineering Science, 142, 318-346.

Sun, Z., Xue, L. F., Wang, S. T., Wang, X. H., and Shi, J. Y. (2016). Single Step Conversion of Cellulose to Levulinic Acid using Temperature-responsive dodecaaluminotungstic Acid Catalysts. Green Chemicals, 18, 742-752.

Upare, P. P., Yoon, J. W., Kim, M. Y., Kang, H. Y., Hwang, D. W., and Chang, J. S. (2013). Chemical Conversion of Biomass-derived Hexose Sugars to Levulinic Acid over Sulfonic Acidfunctionalized Graphene Oxide Catalysts. Green Chemicals, 15, 2935-2943.

Yuan, Z., Long, J., Xia, Y., Zhang, X., Wang, T., and Ma, L. (2016). Production of Levulinic acid from Pennisetum alopecuroides in the Presence of an Acid Catalyst. Bioresources, 11(13). 